\title{
Easing the Burden: \\ Treating Refractory Anxiety
}

\author{
By Jack M. Gorman, MD
}

Treating anxiety disorders can be one of a physician's most gratifying undertakings. For more than 40 years we have had medications, including benzodiazepines and antidepressants, that are highly effective for panic disorder, social anxiety disorder, and generalized anxiety disorder. Antidepressants are also effective for posttraumatic stress disorder and, although less so, also work for obsessive-compulsive disorder (OCD). Over the same timeframe, controlled clinical trials have left no doubt that several forms of psychosocial interventions, most notably cognitive-behavioral therapy, are at least as effective as medication for the treatment of anxiety disorders.

Given this successful track record, is there a need to devote precious research resources to developing methods of treating treatment-resistant anxiety disorders? Our ability to treat schizophrenia, Alzheimer's disease, bipolar disorder, multiple sclerosis, and epilepsy is surely more limited than our ability to treat anxiety disorders and results in a greater number of patients for whom treatments fail.

The treatment-resistant question arises from two important misconceptions. First, while it is true that fear and anxiety are ubiquitous human emotionsindeed they are manifest throughout phylogeny-it is not true that anxiety disorders are easy to treat. Unlike the fear-conditioned rat whose freezing behavior to a stimulus can be extinguished fairly easily, patients with panic disorder, social anxiety disorder, and generalized anxiety disorder cling tenaciously to their worries, avoidant behaviors, and apprehension. Most began their careers as anxious individuals in early childhood and many subsequently develop severe depression, suicidal ideation, substance abuse, and an array of other medical conditions including hypertension, migraine headaches, and coronary artery disease. Anxiety disorders are no less serious for being common and on a continuum with normal behavior.

Second, there are many instances in which conventional therapy fails to bring a patient with an anxiety disorder to full remission. OCD is probably the least tractable anxiety disorder to treatment. Clinical trials of OCD patients often define success as a $30 \%$ improvement, a benchmark reached by $\sim 50 \%$ of patients on active treatment compared with $\sim 0 \%$ receiving no treatment. Response rates for the other anxiety disorders are better, but total remission is induced in a minority of patients and relapse after successful treatment, particularly after the discontinuation of effective medication, is common. Hence, there is a clear need to develop strategies for the many patients with anxiety disorders who do not have sufficiently robust responses to conventional medication and psychotherapy approaches.

Most of the authors in this issue of CNS Spectrums emphasize pharmacologic strategies for treatmentresistant anxiety. While their strategies are certainly interesting, clearly what we need are novel approaches to anxiolytic drug development. We have been stuck with medications that either enhance $\gamma$-aminobutyric acid or block serotonin reuptake for too long. Given the wonderful advances in understanding the basic molecular and cellular biology of fear from preclinical research, it should be possible to advance the field of anti-anxiety medication much more quickly than we have recently witnessed. This will take a sustained effort from funding sources and scientists.

At the same time, psychosocial intervention research must be enhanced. As mentioned earlier, it is now clear that for most anxiety disorders, cognitive-behavioral therapy is a first-line treatment of proven efficacy and durability. Here again, both basic and clinical research should give us even more effective strategies.

The key, of course, is research. To keep research at the forefront, we will have to cast aside the notion that anxiety disorders are somehow not serious enough to warrant scientific attention. We must continue to recognize that solving the treatmentresistant anxiety disorders problem will brighten the lives the millions of people. CNS

Dr. Gorman is the editor of this joumal and Esther and Joseph Klingenstein Professor of Psychiatry and chair of the Department of Psychiatry at Mount Sinai School of Medicine in New York City. 